\title{
Review
}

Dan Wang*

\section{Infection in central venous catheter led by parenteral nutrition of tumor patients}

DOI: $10.2478 / \mathrm{ii}-2018-0005$

Received January 28, 2018; accepted February 06, 2018; published online April 10, 2018

\begin{abstract}
With the wide application of parenteral nutrition (PN) and central venous catheter (CVC) in tumor patients, the relative infection led by CVC has become a common and troublesome hospital infection in clinics. During infusion, PN fluid forms a high-sugar and high-fat microenvironment around CVC, facilitating the adhesion, growth, and diffusion of pathogenic bacteria. This condition forms a bacterial biofilm (BF) consisting of one or several kinds of pathogenic bacteria. Upon formation of the film, pathogens in the BF can resist antibacterial drugs and immune cells, causing repeated infections of bacteria or fungi and endangering the lives of patients. In this article, we summarize the applications of PN, characteristics of CVC infection in tumor patients, mixed BF, and related research methods to provide reference for studies of mixed BF infection of CVC.
\end{abstract}

Keywords: tumor, parenteral nutrition, relative infection of central venous catheter, biofilm

Parenteral nutrition (PN) is the primary means of meeting nutrition demands of patients with esophageal and gastroenteric tumors, tumor cachexia, and long-term endotracheal intubation or in other words, those incapable of eating through the mouth or intestine. In recent years, with the continuous improvement of nutrient solution formulation and infusion technology, tolerance and safety of PNs have improved. However, intestinal dysfunctions [1] caused by long-term PN have significantly increased the incidence of intestinal sepsis; such a dysfunction includes intestinal mucosal atrophy, immune dysfunction, and intestinal bacterial translocation.

Clinical PN patients exhibit susceptibility to dissonance due to long-term application of broad-spectrum antimicrobial agents; especially, patients with malignant tumors feature low autoimmune function. Intestinal flora, such as Escherichia coli, Enterococcus faecalis, Candida albicans, and other bacteria or fungi, pass through the intestinal mucosal barrier and enter the body to cause infection.

Infectious complications of PN are mainly related to catheter-related sepsis [2]. Total PN (TPN) of tumor patients is characterized by long infusion time and high concentration of PN liquid and often requires a central venous catheter (CVC) for infusion. PN fluid forms a high-sugar and high-fat microenvironment around the CVC, facilitating adhesion, growth, and diffusion of pathogenic bacteria in the CVC; a mixedspecies biofilm (BF) comprises one or several kinds of pathogenic bacteria [3].

Once the bacterial BF is formed, the pathogens present can resist antibacterial drugs and immune cells, causing repeated infections by bacteria or fungi. Thus, the film must be often removed from the infected venous catheter. Given the demands for nutrition by patients, a new CVC must be embedded. Therefore, repeated process of catheter-infection-removal-re-catheter not only aggravates medical burden for patients but also contributes to the death of patients.

Our previous research $[4,5]$ suggested that fat emulsions promote bacterial adhesion, germination, growth, and BF formation [6,7]. However, studies have not reported adhesion of fat emulsion on the surface of mixed BFs.

Department of Breast Surgery, The Second Hospital of Jilin University, Changchun 130041, China

*Correspondence: Dan Wang, E-mail: wangdan79@126.com 


\section{Clinical application and complications of PN}

\subsection{Clinical application of PN}

PN refers to supplying a patient with water, calories (sugar and fat emulsion), amino acids (essential and non-essential), vitamins, and electrolytes through the venous pathway. This process is conducted to satisfy the basic nutritional requirements of patients [8] who cannot eat through the gastrointestinal tract and may also be applied to underfed patients to maintain normal nutritional status in intravenous infusion, promote wound healing, slow down weight loss, and ensure continuous growth for sick children. PN has been widely used since its introduction to clinics in the 1960s. In recent years, tolerance and safety of PN have been further improved with continuous improvement of nutrient solution formulation and infusion technology. PN not only provides effective nutritional support for critically ill patients and patients who cannot be ingest through the gastrointestinal tract but has also become an integral part of clinical treatment [9]. Currently, patients who benefit more from PN experience intestinal obstruction, short bowel syndrome, tumor cachexia, severe malnutrition, and metabolic diseases (such as severe burns and severe infections). Patients requiring $\mathrm{PN}$ in the short term correspond to those who have received gastrointestinal operation, those in perioperative period of major surgery, those with intestinal fistula, those who received assisted mechanical ventilator, and those with tumor cachexia.

\subsection{Complications}

Complications of PN include hyperglycemia, hyperlipidemia, hyperosmotic nonketotic acid intoxication coma, hypoglycemia, electrolyte disturbance, liver function injury, and infection [10]. In recent years, numerous studies have shown that long-term usage of PN and TPN can also lead to intestinal dysfunctions [11], such as mucosal atrophy, immune dysfunction, and intestinal bacterial translocation, which are potential risk factors for systemic infection. Improving the function of the intestinal tract benefits tumor malignancy in patients [12]. In view of the abovementioned complications, people have gradually switched from single use of TPN to Enteral Nutrition (EN) + PN. Relevant studies have shown that the combination of EN + PN benefits patients with intestinal function (peristalsis, digestion, and absorption) in terms of recovery, sedimentation reduction in the cholesterol level, improvement in nutritional status and survival rate and effective shortening of patients' hospital stay [13]. For these reasons, adoption of EN is recommended as early as possible.

\section{CVC-associated infection in tumor patients undergoing PN}

During specific treatments, patients will inevitably be embedded with temporary or permanent fixture, such as catheters, tracheal intubation, surgical suture, titanium clip, titanium nail, drainage tube, and venous catheter [14]. Once patients become infected through an embedded material, bacteria and fungi will form bacterial/fungal BFs [15]. BFs comprise colonies of bacteria or fungi attached to the surface of living tissues. $\mathrm{BFs}$ are encased in an extracellular matrix and exhibit definite structure and function. BFs are a unique form of life complex that differs among individuals and dispersed free-state microorganisms and are formed in the long-term evolution of bacterial cells to adapt to the environment [16]. Bacterial BFs can protect bacteria, increase their ability to develop resistance to antibiotics, and render ability to attack the body's immune system. This condition can lead to difficulties in treatment and relapse of infection, which can seriously affect the quality of life of patients and immune function and threaten the life of patients.

A number of studies have shown that tumor cells secrete a variety of immunosuppressive factors [17], such as transformation growth factor- $\beta_{1}$ that evades immune system attacks, prostaglandin $\mathrm{E}_{2}$, vascular endothelial growth factor, interleukin (IL)-10, IL-6, IL-4, and cyclooxygenase-2. These immunosuppressive agents can reduce immunological and humoral immunity of tumor patients through various means, leading 
to low immune function. At the same time, the patient's immune function may be further damaged during surgery, chemotherapy, radiotherapy, and minimally invasive interventional therapy.

Almost all tumor patients must be repeatedly embedded with CVC [18], especially those undergoing surgery and chemotherapy during tumor treatment to provide nutritional support in the long term. Infusion fluid and PN liquid form high-sugar and high-lipid microenvironment around the ducts in the blood vessel cavity, which benefits adhesion, growth, and diffusion of pathogenic bacteria on the surface of CVC and makes the area highly susceptible to bacterial infection. During treatment, a large number of broad-spectrum antibiotics are used, resulting in dissonance of flora and heterotopic conditions of pathogenic bacteria and subsequent formation of mixed bacterial-fungal BFs [3,19]. Once mixed BFs are formed, difficulty in treatment significantly increases compared with single pathogenic bacteria BF, which requires at least a combination of antifungal and bacterial antibiotics. However, the effect is not optimal and further aggravates colony conditions, seriously affecting prognosis of patients.

\section{Mixed BFs}

Mixed bacteria-fungal infections form boundless BFs by competing adhesion sites, space, and nutrients [20]. In terms of coexistence, the organisms can be represented as antagonistic, cooperative, and symbiotic. In formation of mixed bacterial-fungal BFs, the most difficult part of clinical treatment is interaction and synergy of drugs used.

Two kinds of interaction exist between bacteria and fungi: (1) the physical support of surface structures (such as cell wall, mycelium, flagella, and cilia) [21] and (2) through interactions of secretion or autolysis chemicals (such as signaling molecules, extracellular polysaccharide, protein, and DNA and RNA) to complete the exchange and information transmission of genes and metabolites, change antibiotic resistance, adapt to environment pressures, and express virulence genes. Given the complexity of the interaction between bacteria and fungi, scarce information can explain the specific mechanisms, which must be further studied. After fully understanding such interactions, problems of infection, planting, and drug resistance can be potentially eradicated to effectively prevent and treat bacterial-fungal infection.

\section{Effect of PN fluid in the formation of BF}

As nutrient, PN fluid benefits growth of microorganisms and promotes formation of BF. In our preceding study, experimental methods, including crystal violet staining, half quantitative confocal laser scanning microscopy (CLSM), and electron microscopy, were used to study the influence of glucose with different concentrations on BF formation of Staphylococcus epidermidis; results showed that a high concentration of glucose benefits formation of bacterial BF [5]. Fat emulsion injection, another important component of PN, has become a common drug for patients with poor diet and malnutrition. In fat emulsion injection through CVC, high-lipid microenvironment forms in the local tubules and blood, where microorganisms, owing to the nutrients, easily grow. The existence of input materials of CVC causes the microorganisms to easily adhere and form BFs.

Studies have shown that fat emulsion significantly promotes growth of $C$. albicans and formation of hypha [7]. Electron microscopy and CLSM were adopted by Swindell et al. [6] to compare and observe fat emulsion (with different concentrations added to the nutrient solution) and nutrient solution groups; the researchers discovered that in the early stage of BF formation, germination of fat emulsion group and adherence of $C$. albicans increased. In middle and late stages of BF formation, the number of $C$. albicans was more complicated. Germination of fungi serves as an important factor of virulence. Fungal hyphae form an important skeletal component of $\mathrm{BF}$, and experts speculate that fat milk will benefit formation of mixed $C$. albicans-bacteria BFs. However, no studies have reported the formation of mixed bacterial and fungal BFs. 


\section{Identification methods for mixed BFs}

When infected with mixed bacteria, CVC is often used for bacterial culture and bacterial identification. Normally, three working days or longer are required for bacterial infection, including those of anaerobic bacteria. In this process, empirical antibiotic treatment is not only incapable of improving bacteremia of patients but can also actually worsen the disease. At present, fluorescence in situ hybridization (FISH) is the fastest strain identification method for bacteria.

Application of FISH in CVC infection presents advantages, such as being time saving, accurate, and visible via microscopy. However, it is relatively higher in terms of treatment cost [22,23]. According to the common primers used in conservative areas of bacterial 16S rRNA and fungal 18S rRNA, FISH can identify the type of infectious bacteria via fluorescence labeling of different strains and observing via CLSM or fluorescence microscopy. Under normal conditions, the process of obtaining specimens to complete identification totals 2 h. Four steps can be adopted [24]: (1) fixation and penetration of bacteria, (2) hybridization, (3) sample washing, and (4) observation and identification.

Based on the microenvironment of bacterial BFs and studies on bacterial BFs with nutrient solution of different concentrations, we may discover effective methods of preventing BF formation by searching for appropriate infusion speed and concentration of nutrient solution. Some methods may avoid development of a high-sugar and high-lipid microenvironment, which benefits BF formation. Once BF is formed, FISH can be used in the early phase to rapidly and accurately identify the infected strains and develop an effective treatment regimen to control infection.

Acknowledgments: None.

Conflict of interest: The author states no conflicts of interest.

\section{References}

[1] Ralls M.W., Demehri F.R., Feng Y., Woods Ignatoski KM, Teitelbaum D.H., Enteral nutrient deprivation in patients leads to a loss of intestinal epithelial barrier function, Surgery, 2015, 157(4), 732-742.

[2] Ardura M.I., Lewis J., Tansmore J.L., Harp P.L., Dienhart M.C., Balint J.P., Central catheter-associated bloodstream infection reduction with ethanol lock prophylaxis in pediatric intestinal failure: broadening quality improvement initiatives from hospital to home, JAMA Pediatr., 2015, 169(4), 324-331.

[3] Aguilella V.M.J., Valero Z.M.A., Gastalver M.C., Gomis Muñoz P., Moreno Villares J.M., León Sanz M., Incidence of catheter-related infection and associated risk factors in hospitalized patients with parenteral nutrition, Nutr. Hosp., 2012, 27(3), 889-893.

[4] Xu Y.S., Wang X., Xu Y.M., Lei Y.J., He M., Huang Y.C., The effect of blood glucose on the formation of epidermal staphylococcus epidermis on the epidermal staphylococcus epidermis of the tree shrew. Essays Compilation of Chinese Medical Association National Endocrinology Conference, Suzhou China. 2011: 2.

[5] Xu Y.S., Wang X., Xu Y.M., Lei Y.J., He M., Huang Y.C., The effects of blood glucose on the removal of epidermal staphylococcus epidermal staphylococcus epidermis and the adhesion of implantable materials, CRTER Clin. Rehabil., 2011, 15(51), 9570-9574.

[6] Swindell K., Lattif A.A., Chandra J., Mukherjee P.K., Ghannoum M.A., Parenteral lipid emulsion induces germination of Candida albicans and increases biofilm formation on medical catheter surfaces, J. Infect. Dis., 2009, 200(3), 473-480.

[7] Wanten G.J., Netea M.G., Naber T.H., Curfs J.H., Jacobs L.E., Verver-Jansen T.J., et al., Parenteral administration of medium- but not long-chain lipid emulsions may increase the risk for infections by Candida albicans, Infect. Immun., 2002, 70(11), 6471-6474.

[8] Riskin A., Hartman C., Shamir R., Parenteral nutrition in very low birth weight preterm infants, Isr. Med. Assoc. J., 2015, 17(5), 310-315.

[9] Torgersen Z., Balters M., Perioperative nutrition, Surg. Clin. North Am., 2015, 95(2), 255-267.

[10] Cheng Y., Li J., The research progress of the prevention and treatment to the related complications of parenteral nutrition of premature infant, Parenteral Enteral Nutr., 2015, 22(1), 53-56.

[11] Jakobsen M.S., Jørgensen M.H., Husby S., Andersen L., Jeppesen P.B., Low-fat, high-carbohydrate parenteral nutrition (PN) may potentially reverse liver disease in long-term PN-dependent infants, Dig. Dis. Sci., 2015, 60(1), 252-259. 
[12] Klein G.L., Petschow B.W., Shaw A.L., Weaver E., Gut barrier dysfunction and microbial translocation in cancer cachexia: a new therapeutic target, Curr. Opin. Support Palliat. Care, 2013, 7(4), 361-367.

[13] Nomura E., Lee S.W., Kawai M., Hara H., Nabeshima K., Nakamura K., et al., Comparison between early enteral feeding with a transnasal tube and parenteral nutrition after total gastrectomy for gastric cancer, Hepatogastroenterology, 2015, 62(138), 536-539.

[14] Chen Y., Lei Y.J., Huang Y.C., Rao Z.M., Ye L.H., Zhao G.Q., et al., The effect of aggregation related protein gene and transformation growth factor $\beta 1$ on the biofilm formation of epidermal staphylococcus epidermis of lung cancer, Chin. J. Lung Cancer, 2014, 18(4), 308-314.

[15] Lei Y.J., Huang Y.C., Yang L., Guo F.L., Zhao G.Q., Xu Y.S., et al., The relationship between the biological material related infection of lung cancer patients and $\beta 1$ level of peripheral blood transformation, CRTER Clin. Rehabil., 2011, 15(47), 8907-8910.

[16] Walraven C.J., Lee S.A., Antifungal lock therapy, Antimicrob. Agents Chemother., 2013, 57(1), 1-8.

[17] Kim E.S., Kim M.S., Moon A., Transforming growth factor (TGF)-beta in conjunction with H-ras activation promotes malignant progression of MCF10A breast epithelial cells, Cytokine, 2005, 29(2), 84-91.

[18] Lei Y.J., Huang Y.C., Yang L., Guo F.L., Xu Y.S., Ye L.H., et al., The relationship of icaA, icaD expression and transformation growth factor $\beta 1$ and biofilm in patients with lung cancer patients with central venous catheter, CRTER, 2012, 12(19), 2158-2162.

[19] Xu Y.S., Ye L.H., Huang Y.C., Le L.J., Zhang L., The effect of high concentration glucose on the surface formation of epidermal Staphylococcus aureus on the surface of biomaterials, CRTER Clin, Rehabil., 2010, 14(51), 9609-9612.

[20] Polke M., Hube B., Jacobsen I.D., Candida survival strategies, Adv. Appl. Microbiol., 2015, 91(6), 139-235.

[21] Chen Y., Wang X.Y., Zhao G.Q., Lei Y.J., Ye L.H., Huang Q.B., et al., The survey of White Candida albicans - epidermal staphylococcus epidermal staphylococcus mixed biofilm structure, Chin. J. Rehabil. Reconstr. Surg., 2015, 29(5), 609-614.

[22] Carretto E., Bardaro M., Russello G., Mirra M., Zuelli C., Barbarini D., Comparison of the Staphylococcus QuickFISH BC test with the tube coagulase test performed on positive blood cultures for evaluation and application in a clinical routine setting, J. Clin. Microbiol., 2013, 51(1), 131-135.

[23] Harris D.M., Hata D.J., Rapid identification of bacteria and Candida using PNA-FISH from blood and peritoneal fluid cultures: a retrospective clinical study, Ann. Clin. Microbiol. Antimicrob., 2013, 12(1), 2.

[24] Malic S., Hill K.E., Hayes A., Percival S.L., Thomas D.W., Williams D.W., Detection and identification of specific bacteria in wound biofilms using peptide nucleic acid fluorescent in situ hybridization (PNA fish), Microbiology, 2009, 155(Pt 8), 2603-2611. 